\title{
DJ-1 upregulates anti-oxidant enzymes and attenuates hypoxia/re-oxygenation-induced oxidative stress by activation of the nuclear factor erythroid 2-like 2 signaling pathway
}

\author{
YU-FENG YAN $^{1 *}$, WEN-JIE YANG ${ }^{1 *}$, QIANG XU ${ }^{2}$, HE-PING CHEN $^{1}$, XIAO-SHAN HUANG ${ }^{1}$, \\ LING-YU QIU ${ }^{1}$, ZHANG-PING LIAO ${ }^{1}$ and QI-REN HUANG ${ }^{1}$ \\ ${ }^{1}$ Key Laboratory of Basic Pharmacology, School of Pharmaceutical Science, Nanchang University; \\ ${ }^{2}$ Jiangxi Nursing Vocational and Technical College, Nanchang, Jiangxi 330006, P.R. China
}

Received September 3, 2014; Accepted May 15, 2015

DOI: $10.3892 / \mathrm{mmr} .2015 .3947$

\begin{abstract}
DJ-1 protein, as a multifunctional intracellular protein, has an important role in transcriptional regulation and anti-oxidant stress. A recent study by our group showed that DJ-1 can regulate the expression of certain anti-oxidant enzymes and attenuate hypoxia/re-oxygenation (H/R)-induced oxidative stress in the cardiomyocyte cell line H9c2; however, the detailed molecular mechanisms have remained to be elucidated. Nuclear factor erythroid 2-like 2 (Nrf2) is an essential transcription factor that regulates the expression of several anti-oxidant genes via binding to the anti-oxidant response element (ARE). The present study investigated whether activation of the Nrf2 pathway is responsible for the induction of anti-oxidative enzymes by DJ-1 and contributes to the protective functions of DJ-1 against H/R-induced oxidative stress in $\mathrm{H} 9 \mathrm{c} 2$ cells. The results demonstrated that DJ-1-overexpressing H9c2 cells exhibited anti-oxidant enzymes, including manganese superoxide dismutase, catalase and glutathione peroxidase, to a greater extent and were more resistant to H/R-induced oxidative stress compared with native cells, whereas DJ-1 knockdown suppressed the induction of these enzymes and further augmented the oxidative stress injury. Determination of the importance of Nrf2 in DJ-1-mediated anti-oxidant enzymes induction and cytoprotection against oxidative stress induced by $\mathrm{H} / \mathrm{R}$ showed that overexpression of DJ-1 promoted the dissociation of Nrf2 from its cytoplasmic inhibitor Keap1, resulting in enhanced levels of nuclear translocation, ARE-binding and transcriptional activity of Nrf2. Of
\end{abstract}

Correspondence to: Dr He-Ping Chen, Key Laboratory of Basic Pharmacology, School of Pharmaceutical Science, 461 Bayi Road, Nanchang University, Nanchang, Jiangxi 330006, P.R. China

E-mail: chenheping69@hotmail.com

*Contributed equally

Key words: DJ-1, nuclear factor erythroid 2-like 2, anti-oxidative enzymes, hypoxia/re-oxygenation, oxidative stress note, Nrf2 knockdown abolished the DJ-1-mediated induction of anti-oxidant enzymes and cytoprotection against oxidative stress induced by H/R. In conclusion, these findings indicated that activation of the Nrf2 pathway is a critical mechanism by which DJ-1 upregulates anti-oxidative enzymes and attenuates $\mathrm{H} / \mathrm{R}$-induced oxidative stress in $\mathrm{H} 9 \mathrm{c} 2$ cells.

\section{Introduction}

Oxidative stress is known to have an important role in the pathogenesis of certain cardiovascular diseases, including atherosclerosis, myocardial ischemia-reperfusion injury and chronic heart failure (1). Endogenous anti-oxidant systems include a number of proteins or enzymes, e.g., superoxide dismutase (SOD), catalase (CAT) and glutathione peroxidase (GPx), as well as non-enzymatic, low-molecular-weight anti-oxidant compounds, including glutathione and ascorbic acid, which constitute the first line of defence against oxidative stress and maintain the redox environment of the body (2). Mounting evidence indicates that stimulation of endogenous anti-oxidant systems is an important strategy for achieving cardioprotection against ischemia-reperfusion injury, and is also one of essential mechanisms of anti-oxidative stress effect of certain proteins $(3,4)$.

DJ-1, a ubiquitously expressed and highly conserved intracellular protein, was originally discovered in 1997 as a novel oncogene product that can transform mouse NIH3T3 cells in combination with H-Ras or c-Myc (5). Subsequent studies demonstrated that DJ-1 has multiple functions and is involved in diverse cellular processes ranging from cellular transformation, transcriptional regulation and anti-oxidative stress response to control of male infertility (6-10). A recent study by our group investigated the potential role of DJ-1 in the response of $\mathrm{H} 9 \mathrm{c} 2$ cells to oxidative stress induced by hypoxia/re-oxygenation $(\mathrm{H} / \mathrm{R})$ by using gene transfer strategies and firstly found that DJ-1 can upregulate the expression of certain anti-oxidant enzymes (MnSOD, CAT and GPx) and attenuate H/R-induced oxidative stress in H9c2 cells (11). However, the detailed molecular mechanisms by which DJ-1 upregulates these anti-oxidant enzymes have remained to be fully elucidated. 
Nuclear factor erythroid 2-like 2 (Nrf2), the central transcription factor, regulates multiple lines of cellular anti-oxidant systems, which is important in the protection of cells against oxidative stress (12-14). Under basal, non-activated conditions, $\mathrm{Nrf} 2$ is bound to Kelch-like ECH-associated protein-1 (Keap1) in the cytoplasm and is degraded through the ubiquitin-proteasome pathway $(12,15)$. However, once activated, Nrf2 dissociates from its cytoplasmic inhibitor Keap1 and translocates to the nucleus, where it binds to conserved anti-oxidant response elements (ARE) and transactivates the expression of ARE-dependent genes, including MnSOD, CAT, and GPx, to attenuate cellular oxidative stress $(13,16,17)$.

Of note, Clements et al (18) reported that DJ-1 is indispensable for Nrf2 stabilization in primary mouse embryonic fibroblasts, as supported by the fact that disruption of DJ-1 decreased Nrf2 protein stability, whereas overexpression of DJ-1 restored protein stability by promoting Nrf2 disassociation from Keap1 and thus escaping ubiquitin-dependent proteasomal degradation during oxidative stress (18). These results implied that the anti-oxidant effects of DJ-1 may be mediated via the Nrf2 pathway. Accordingly, based on these studies, the present study hypothesized that activation of the Nrf2 pathway may also be responsible for the induction of anti-oxidative enzymes by DJ-1 and contribute to the protective functions of DJ-1 against H/R-induced oxidative stress in H9c2 cells.

To investigate this hypothesis, the present study used DJ-1/Nrf2-knockdown or -overexpressing H9c2 cardiomyocyte cells and assessed levels of anti-oxidant enzymes, as well as indicators of oxidative stress and cell proliferation following H/R. Furthermore, a luciferase reporter assay as well as immunoprecipitation (IP) and immunoblotting (IB) were performed to investigate the association between Nrf2 and Keap1.

\section{Materials and methods}

Chemicals and reagents. Cell culture media and supplements were obtained from Invitrogen Life Technologies (Carlsbad, CA, USA). 5(6)-carboxy-2',7'-dichlorofluoresceindiacetate (cDCFH-DA) was purchased from Molecular Probes Inc. (Thermo Fisher Scientific, Waltham, MA, USA). A malondialdehyde (MDA) assay kit was obtained from Nanjing Jiancheng Bioengineering Institute (Nanjing, China). Antibodies against DJ-1 (N-20; cat. no. sc-27004; goat; pAb; 1:1,000), Nrf2 (C-20; cat. no. sc-722; rabbit; pAb; 1:1,000), Keap1 (H-190; cat. no. sc-33569; rabbit; pAb; 1:1,000), MnSOD (N-20; cat. no. sc-18503; goat; pAb; 1:500), CAT (N-17; cat. no. sc-34280; goat; pAb; 1:500), GPX (N-20; cat. no. sc-22145; goat; pAb; 1:300), lamin B1 (A-11; cat. no. sc-377000; mouse; mAb; 1:1,000), and $\beta$-Actin (I-19; cat. no. sc-1616; goat; pAb; 1:1,000) were purchased from Santa Cruz Biotechnology (Santa Cruz, CA, USA); anti-Flag (cat. no. F2555; rabbit; $\mathrm{mAb}, 1: 1,000$ ) and anti-tubulin (cat. no. T8203; mouse; mAb; 1:1,000) were purchased from Sigma-Aldrich (St. Louis, MO, USA). All primary antibodies were incubated overnight at $4^{\circ} \mathrm{C}$. Horseradish peroxidase (HRP)-conjugated secondary antibodies (1:5,000; goat anti-mouse IgG-HRP; cat. no. sc-2005, rabbit anti-goat
IgG-HRP; cat. no. sc-2768, and goat anti-rabbit IgG-HRP; cat. no. sc-2004) were used at room temperature for $1 \mathrm{~h}$. All other chemicals were also purchased from Sigma-Aldrich, unless otherwise stated.

Cell culture. H9c2, a clonal cell line derived from an embryonic rat heart, was obtained from the American Type Culture Collection (CRL-1446; Manassas, VA, USA) and were maintained in Dulbecco's modified Eagle's medium (DMEM) supplemented with $10 \%(\mathrm{v} / \mathrm{v})$ heat-inactivated fetal bovine serum, $10 \mathrm{mM} \mathrm{L-Glu}$ and $5 \mathrm{mg} / \mathrm{ml}$ penicillin/streptomycin (complete medium) in a humidified atmosphere of $95 \%$ air and $5 \% \mathrm{CO}_{2}$ at $37^{\circ} \mathrm{C}$. The medium was replaced every other day and the cells were seeded in six-well plates at a density of $1 \times 10^{6}$ cells/well.

DJ-1 expression vector construction and transfection. The rat DJ-1 gene expression vector was constructed as previously described (11). Briefly, to obtain the coding region of rat wild-type DJ-1 cDNA, oligonucleotide primers complementary to the $5^{\prime}$ and $3^{\prime}$ ends of DJ-1 were designed, which incorporated the restriction sites EcoR I and Kpn I: Primer sense, 5'-CCGGAATTCAATGGCATCCAAAAG AGC; and primer anti-sense, 5'-CGGGGTACCCTAGTCTTT GAGAACAAGCG. The specific primers were designed and synthesized by Shanghai Jikai Gene Chemical Co., Ltd, (Shanghai, China). Total RNA was extracted from H9c2 cells using TRIzol ${ }^{\circledR}$ reagent (Invitrogen Life Technologies, Carlsbad, CA, USA) according to the manufacturer's instructions. DJ-1 cDNA was amplified using a Multiplex PCR kit (Takara Bio, Inc., Shiga, Japan) by a PCR reactor (Bio-Rad Laboratories, Inc., Hercules, CA, USA). The PCR conditions were as follows: Initial denaturation at $95^{\circ} \mathrm{C}$ for $5 \mathrm{~min}$; 30 cycles of denaturation at $95^{\circ} \mathrm{C}$ for $30 \mathrm{sec}$, annealing at $57^{\circ} \mathrm{C}$ for $1 \mathrm{~min}$ and elongation at $72^{\circ} \mathrm{C}$ for $1 \mathrm{~min}$ and a final extension at $72^{\circ} \mathrm{C}$ for $5 \mathrm{~min}$. Polymerase chain reaction (PCR) fragments were separated by electrophoresis on a $1.5 \%$ agarose gel (Sigma-Aldrich) and visualized with ethidium bromide (Beyotime Institute of Biotechnology, Haimen, China). PCR products were digested with EcoR I and Kpn I (Takara Bio, Inc.) and analyzed by $12 \%$ agarose gel electrophoresis. Bands of the expected length were excised from the gel and ligated into the mammalian expression vector pFlag-CMV-4 (Sigma-Aldrich), which had been digested with EcoR I and $K p n$ I. Subsequently, the generated construct was confirmed by sequencing and further referred to as pFlag-DJ-1. pFlag-DJ-1 or empty vector pFlag were transfected into H9c2 cells using Lipofectamine 2000 reagent (Invitrogen Life Technologies) according to the manufacturer's instructions. DNA sequencing was performed by Shanghai Jikai Gene Chemical Co., Ltd.

Small interfering RNA and transfection. The small interfering RNA (siRNA) oligonucleotides targeting rat DJ-1 or Nrf2 were purchased from Ambion Inc. (Thermo Fisher Scientific). A negative control siRNA (NC siRNA) was included to monitor non-specific effects. These siRNAs were transfected into H9c2 cells using Lipofectamine 2000 reagent. $24 \mathrm{~h}$ after transfection, western blot analysis was performed to examine the knockdown of the target proteins. 
Cellular models of $H / R . \mathrm{H} / \mathrm{R}$ was conducted as previously described $(11,19)$. Briefly, hypoxia was achieved by incubating the cells for $2 \mathrm{~h}$ in an airtight chamber in which $\mathrm{O}_{2}$ was replaced by $\mathrm{N}_{2}$ using glucose-free Tyrode's solution that contains $139 \mathrm{mM} \mathrm{NaCl}, 4.7 \mathrm{mM} \mathrm{KCl}, 0.5 \mathrm{mM} \mathrm{MgCl}, 1.0 \mathrm{mM}$ $\mathrm{CaCl}_{2}$ and $5 \mathrm{mM}$ HEPES, $\mathrm{pH} 7.4$, at $37^{\circ} \mathrm{C}$. After incubation under hypoxic conditions, the cells were provided with fresh medium and then cultured under normoxic conditions $(95 \%$ air-5\% $\mathrm{CO}_{2}, 37^{\circ} \mathrm{C}$ ) for $60 \mathrm{~min}$ for re-oxygenation.

Quantification of cell damage. Cell damage was determined by measuring cell viability and the release of lactate dehydrogenase (LDH) into the cell culture medium. The release of LDH was quantified using the CytoTox-ONE ${ }^{\mathrm{TM}}$ Homogenous Membrane Integrity Assay (Promega Corp., Madison, WI, USA) according to the manufacturer's instructions. Cell viability was determined using an MTT assay based on the formation of formazan from MTT by metabolically active cells. Briefly, the cells were seeded into 96-well plates at $1 \times 10^{5}$ cells/well. Following treatment, cells were washed with warm phosphate-buffered saline (PBS) and incubated with $0.5 \mathrm{mg} / \mathrm{ml}$ MTT in PBS for $4 \mathrm{~h}$ at $37^{\circ} \mathrm{C}$. The reaction was stopped by the addition of $150 \mu \mathrm{l}$ diphenylamine solution, and the absorbance of the blue formazan derivative read at $570 \mathrm{~nm}$ using a microplate reader (680; Bio-Rad Laboratories, Inc.).

Detection of reactive oxygen species (ROS) generation. The production of ROS, as an index of oxidative stress, was determined using the cell-permeable probe cDCFH-DA, which is cleaved by cellular esterases to non-fluorescent 2',7'-dichlorofluorescin (DCFH) and oxidized by intracellular ROS to the fluorescent product dichlorofluorescein (DCF). After the indicated treatments, the cells were harvested and washed with cold PBS, following by incubation with $10 \mathrm{mM}$ of cDCFH-DA at $37^{\circ} \mathrm{C}$ for $20 \mathrm{~min}$. DCF fluorescence was then monitored by flow cytometry (FACSCalibur; BD Biosciences, Franklin Lakes, NJ, USA) at wavelengths of $495 \mathrm{~nm}$ (excitation) and $525 \mathrm{~nm}$ (emission). The generation of ROS was expressed as the mean fluorescence intensity of 10,000 cells determined in each sample.

Measurement of MDA content. MDA, a breakdown product of lipid peroxidation, is another oxidative stress marker and truly reflects the development of oxidative stress in a biological system. After treatment, cells were harvested and lysed in $100 \mu 1$ lysis buffer containing 1\% Triton X-100, $10 \mathrm{mM}$ Tris, $\mathrm{pH}$ 7.6, $50 \mathrm{mM} \mathrm{NaCl}, 0.1 \%$ bovine serum albumin (BSA), $1 \mathrm{mM}$ phenylmethanesulfonylfluoride, $1 \%$ aprotinin, $5 \mathrm{mM}$ EDTA, $50 \mathrm{mM} \mathrm{NaF}, 0.1 \%$ 2-mercaptoethanol, $5 \mathrm{mM}$ phenylarsine oxide and $100 \mathrm{mM}$ sodium orthovanadate by three cycles of freezing/thawing. After centrifugation at 13,000 xg for $10 \mathrm{~min}$ at $4^{\circ} \mathrm{C}$, the supernatants were subjected to the cellular MDA content assay using the corresponding detection kit according to the manufacturer's instructions. The results were normalized to the total protein levels, as measured by the Lowry method using a Bio-Rad detergent-compatible (DC) protein assay kit II, (Bio-Rad Laboratories, Inc.).

Western blot analysis. Cytoplasmic and nuclear proteins were extracted using NE-PER Nuclear and Cytoplasmic Extraction
Reagents kit (Pierce, Rockford, IL, USA) according to the manufacturer's instructions. The protein content was determined by the Lowry method using a DC protein assay kit. IB analysis was performed as described previously $(11,19)$. In brief, equal amounts of proteins were resolved on $12 \%$ SDS-PAGE gels (Sigma-Aldrich) and transferred onto nitrocellulose membrane (Schleicher and Schuell, Keene, NH, USA). After blocking with 5\% non-fat milk, the blots were probed using primary antibodies against DJ-1 (dilution, 1:1,000), Flag (1:1,000), Nrf2 (1:1,000), MnSOD (1:500), CAT (1:500), GXp (1:300), lamin-B1 (1:1,000), tubulin $(1: 1,000)$, lamin-B1 $(1: 1,000)$ and $\beta$-actin $(1: 1,000)$. Appropriate secondary antibodies conjugated with horseradish peroxidase immunoglobulin $(\mathrm{Ig}) \mathrm{G}$ were used for chemiluminescence detection. An enhanced chemiluminescence kit was used (Pierce). The wet transfer system was used (Bio-Rad Laboratories, Inc.) and the X-ray film was purchased from Kodak (Rochester, NY, USA). The band intensities were determined using Quantity One ${ }^{\circledR}$ image analysis software version 4.62 (Bio-Rad Laboratories, Inc.).

Co-IP assays. To assess the interaction between Nrf2 and Keap1, whole-cell lysates containing $0.5 \mathrm{mg}$ protein were pre-cleared with protein G-agarose (Roche Applied Science, Basel, Switzerland) at $4^{\circ} \mathrm{C}$ for $3 \mathrm{~h}$. Subsequently, IP was performed overnight with $1 \mu \mathrm{g}$ anti-Nrf2 antibody at $4^{\circ} \mathrm{C}$ followed by a 4-h incubation with $30 \mu \mathrm{l}$ protein-G agarose. After washing, the IP complexes were subjected to IB with anti-Nrf2 or anti-Keap 1 antibodies as described above in the western blot analysis protocol.

Chromatin IP (ChIP) assay. To evaluate the ARE-binding activity of Nrf2, a ChIP assay was performed by using the commercial ChIP-IT kit (Active Motif, Carlsbad, CA, USA) as previously described (19). Briefly, cells were cross-linked by adding formaldehyde, lysed and sonicated. Lysates were immunoprecipitated using Nrf2 antibodies, proteins were digested with proteinase $\mathrm{K}$ and ChIP-enriched DNA was subjected to PCR using the following primers: MnSOD-ARE, forward 5'-CTGAGGGCAAGAGAAAAGAGAT-3' and reverse 5'-TACCCCTAAGTGAGTCCATTGAT-3'; GPx-ARE, forward 5'-CGGGACCCTGAGATTTAGA-3' and reverse 5'-CGAGCAGCAGACATACTG-3'; CAT-ARE, forward 5'-CCAGATTACATTCCTGATTCC-3' and reverse 5'-CGT GGTCACTATGCTGTC-3'. IgG was used as a negative control for IP with anti-Nrf2 to verify the specificity. 10\% of the chromatin DNA used for IP was similarly subjected to PCR analysis and designated as input. PCR products were quantified using ImageJ software version 2.1.4.7 (National Institutes of Health, Bethesda, MD, USA). Relative enrichment was calculated as the difference between Nrf2 antibody and normal IgG signals that were normalized to the respective input signals.

Luciferase reporter assays. The transcriptional activity of Nrf2 was tested by a reporter gene assay. An ARE reporter comprised of tandem repeats of the ARE transcriptional response element upstream of firefly luciferase (SA Biosciences, Frederick, MD) and a renilla luciferase plasmid containing a cytomegalovirus promoter (as an internal control) were used. Transfections of H9c2 cells were performed with 

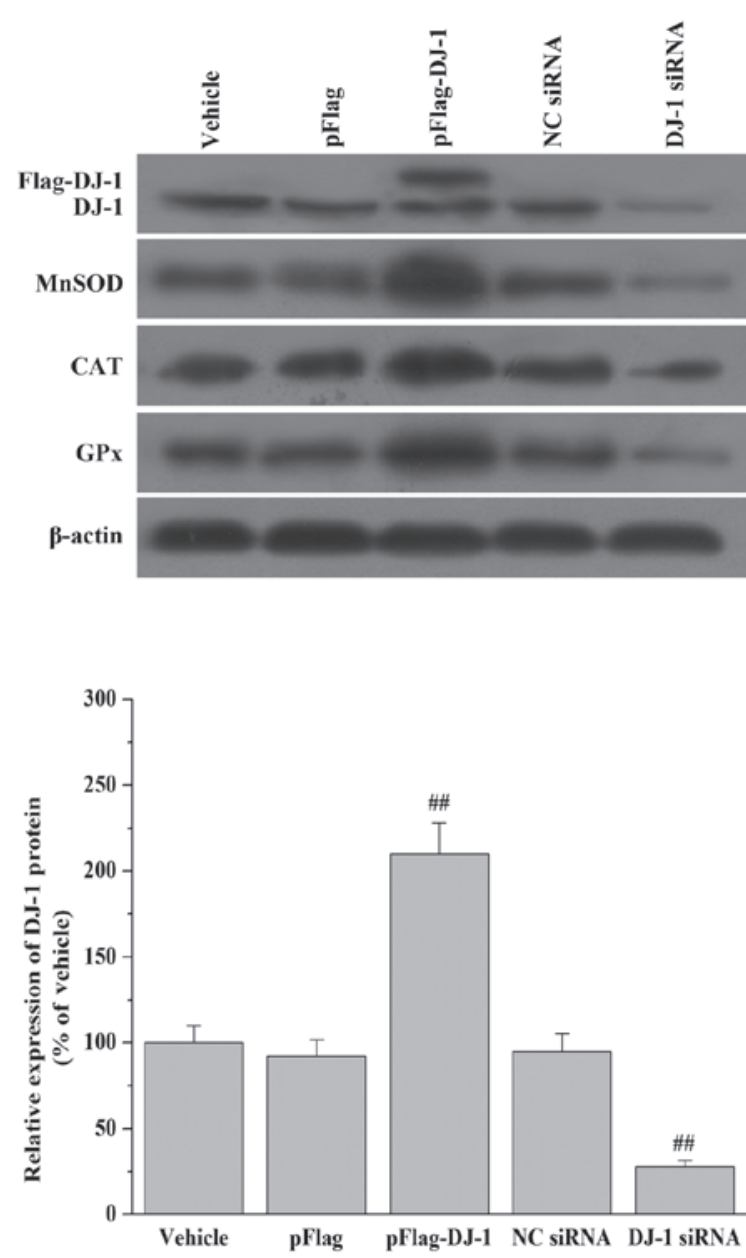
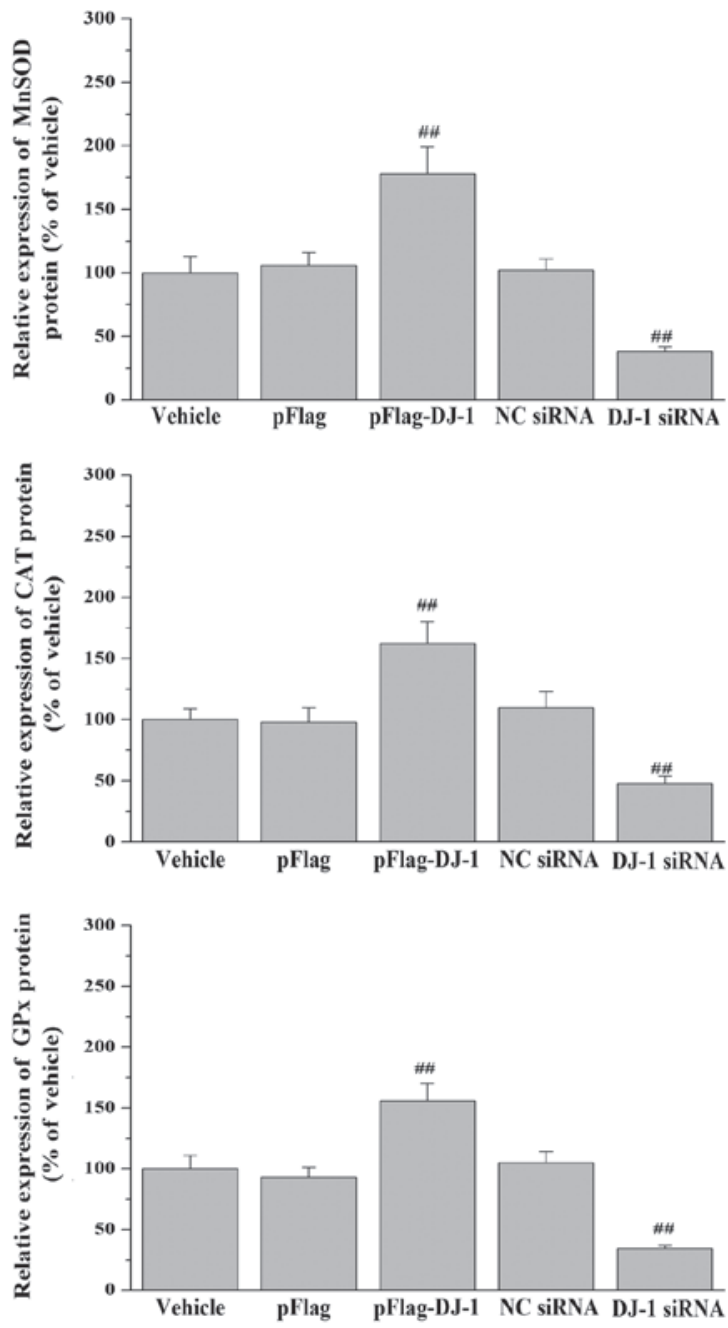

Figure 1. Role of DJ-1 in the regulation of the expression of the anti-oxidant enzymes MnSOD, GPx and CAT. H9c2 cells were transiently transfected with or without NC or nuclear factor erythroid 2-like 2 siRNA, or pFlag-DJ-1 or its empty control vector pFlag for 24 h. Levels of DJ-1 and anti-oxidant enzymes MnSOD, GPx and CAT were examined by western blot analysis. $\beta$-actin was used as an internal control. A representative blot of each experiment is shown. Blots were quantified using densitometric analysis and values are expressed as the mean \pm standard error of the mean of four independent experiments. ${ }^{\# \#} \mathrm{P}<0.01$ vs. vehicle group. siRNA, small interfering RNA; SOD, superoxide dismutase; GPx, glutathione peroxidase; CAT, catalase; NC, negative control.

Lipofectamine 2000 reagent according to the manufacturer's instructions. Firefly and renilla luciferase activities were assayed using the Dual Luciferase ${ }^{\circledR}$ Assay System (Promega Corp.) and results were expressed as firefly luciferase activity normalized to renilla luciferase activity.

Statistical analysis. Values are expressed as the mean \pm standard error of the mean. An unpaired t-test was used to compare between groups. Multiple group means were compared by analysis of variance followed by a least significant difference post hoc test. Statistical calculations were performed using SPSS 11.0 software (SPSS, Inc., Chicago, IL, USA). Differences with a two-tailed $\mathrm{P}<0.05$ were considered statistically significant.

\section{Results}

DJ-1 induces anti-oxidant enzymes and attenuates $H / R$-induced oxidative stress in H9c2 cells. As an initial experiment toward determining the role of DJ-1 in the regulation of cellular anti-oxidant enzymes and attenuation of oxidative stress induced by $\mathrm{H} / \mathrm{R}, \mathrm{H} 9 \mathrm{c} 2$ cells were transiently transfected with pFlag-DJ-1 or DJ-1 siRNA for $24 \mathrm{~h}$ and then subjected to H/R. As shown in Fig. 1, the transfection of pFlag-DJ-1 markedly increased DJ-1 levels concomitant with an obvious elevation in MnSOD, CAT and GPx expression in $\mathrm{H} 9 \mathrm{c} 2$ cells. By contrast, empty vector pFlag did not affect the expression of these proteins. In addition, 24-h transfection of pFlag-DJ-1 markedly attenuated H/R-induced viability loss (Fig. 2A), LDH release (Fig. 2B) as well as elevation of ROS (Fig. 2C) and MDA (Fig. 2D) content, indicating that DJ-1-overexpressing H9c2 cells were more resistant to H/R-induced oxidative stress. Conversely, in DJ-1 siRNA-transfected H9c2 cells, knockdown of DJ-1 significantly reduced endogenous MnSOD, CAT and GPx protein levels (Fig. 1). Concomitantly, these DJ-1-deficient cells were more susceptible to H/R-induced oxidative stress compared with control cells (Fig. 2), which was in agreement with the results for DJ-1-overexpressing cells. Overall, these findings demonstrated that DJ-1 can induce the expression of anti-oxidant enzymes and attenuate $\mathrm{H} / \mathrm{R}$-induced oxidative stress in $\mathrm{H} 9 \mathrm{c} 2$ cells. 

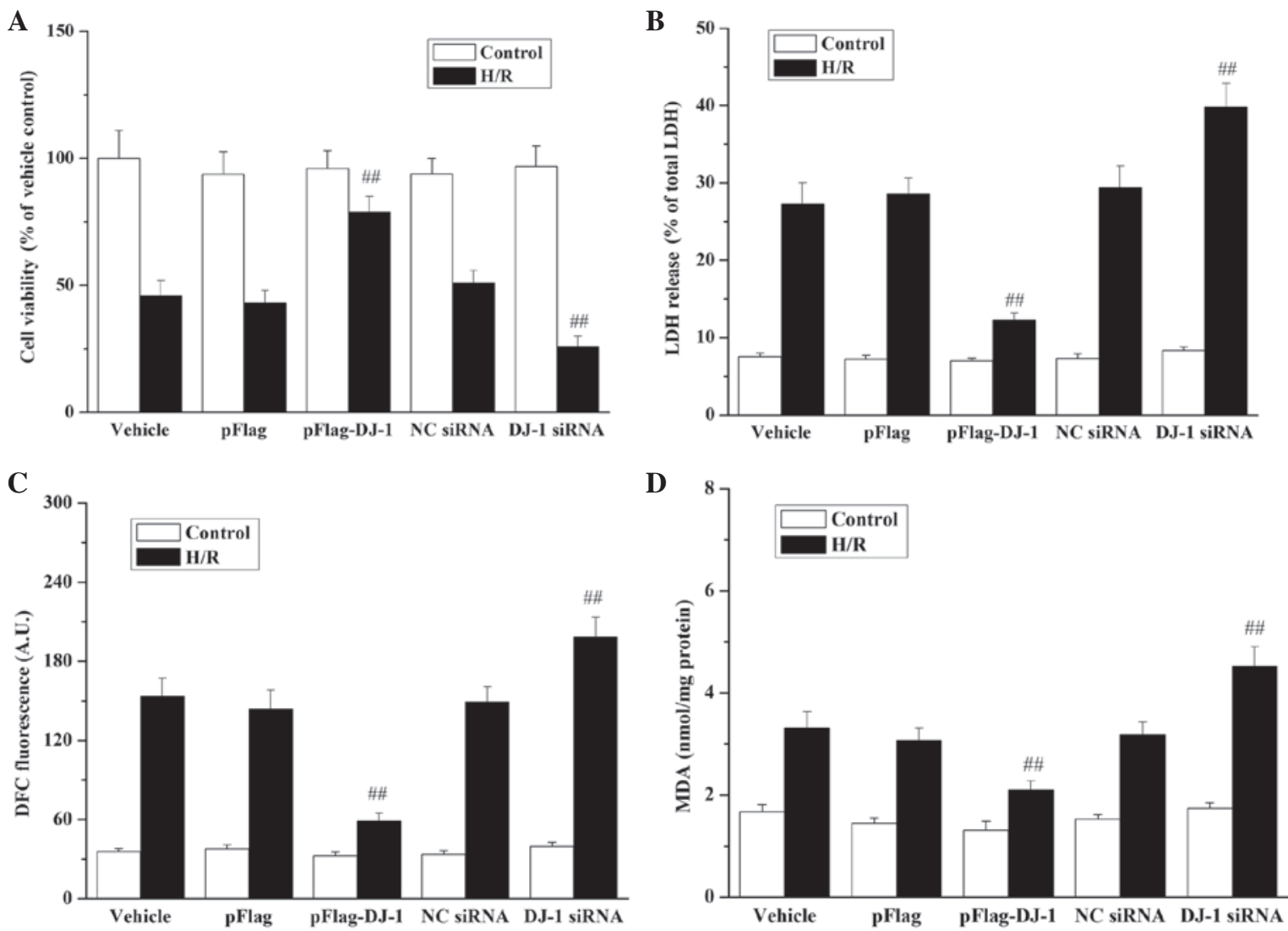

Figure 2. Effects of DJ-1 on H/R-induced oxidative stress injury in H9c2 cells. H9c2 cells were transiently transfected with or without NC or nuclear factor erythroid 2-like 2 siRNA, or pFlag-DJ-1 or its empty control vector pFlag for $24 \mathrm{~h}$ followed by H/R. Subsequently, cell damage was identified by (A) cell viability and (B) LDH release assays, and oxidative stress was assessed by quantifying the (C) ROS and (D) MDA content. Values are expressed as the mean \pm standard error of the mean of four independent experiments. ${ }^{\# \#} \mathrm{P}<0.01$ vs. vehicle $+\mathrm{H} / \mathrm{R}$ group. $\mathrm{H} / \mathrm{R}$, hypoxia/re-oxygenation; MDA, malondialdehyde; ROS, reactive oxygen species; LDH, lactate dehydrogenase; NC, negative control; siRNA, small interfering RNA; DFC, 2',7'-dichlorofluorescein.

DJ-1 promotes the activation of the Nrf2 pathway in $H 9 c 2$ cells. The present study next focused on the underlying mechanisms by which DJ-1 upregulates anti-oxidative enzymes and attenuates H/R-induced oxidative stress in H9c2 cells. Considering that DJ-1 has been suggested to increase Nrf2 stability (18), whereas the Nrf2 pathway has been shown to have a critical role in regulation of anti-oxidative enzymes and in cell survival under stress conditions (12-14), the present study hypothesized that activation of the Nrf2 pathway may be responsible for the induction of anti-oxidative enzymes by DJ-1 and contribute to the protective functions of DJ-1 against H/R-induced oxidative stress. To investigate this putative mechanism, the present study first examined the effects of DJ-1 on the Keap1 - Nrf2 interaction and subsequent nuclear translocation, ARE-binding and transcriptional activity of Nrf2 in H9c2 cells. As shown in Fig. 3, in transfected pFlag-DJ-1 H9c2 cells, DJ-1 overexpression promoted Nrf2 - Keap1 dissociation (Fig. 3A) and resulted in increased nuclear translocation (Fig. 3B), ARE-binding (Fig. 3C) and transcriptional activity (Fig. 3D) of Nrf2. However, in DJ-1-siRNA-transfected H9c2 cells, knockdown of DJ-1 exhibited inhibitory effects on the Keap1 - Nrf2 dissociation (Fig. 3A) as well as on the subsequent nuclear translocation (Fig. 3B), ARE-binding (Fig. 3C) and transcriptional activity (Fig. 3D) of Nrf2. These results indicated that DJ-1 has an obligatory role in the activation of the Nrf2 pathway in H9c2 cells.
Activation of the Nrf2 pathway is required for DJ-1-mediated induction of anti-oxidative enzymes. The present study put further emphasis on the correlation between the activation of Nrf2 pathway and the DJ-1-mediated induction of anti-oxidative enzymes, and attempted to elucidate whether it is an accompanying or a cause-effect phenomenon. For this, the impact of Nrf2 knockdown on the DJ-1-mediated induction of anti-oxidative enzymes was investigated. H9c2 cells were transfected with pFlag-DJ-1 or empty vector pFlag for $24 \mathrm{~h}$ and subsequently with negative control (NC) or Nrf2 siRNA for $24 \mathrm{~h}$. As shown in Fig. 4, DJ-1 overexpression upregulated anti-oxidative enzymes (MnSOD, CAT and GPx) consistent with the results shown in Fig. 1, whereas simultaneous knockdown of Nrf2 significantly attenuated DJ-1-mediated induction of anti-oxidative enzymes (Fig. 4). These results indicated that activation of the Nrf2 pathway is required for DJ-1-mediated induction of anti-oxidative enzymes.

Activation of the Nrf2 pathway is required for DJ-1-mediated cytoprotection against oxidative stress induced by $H / R$. The abovementioned results strongly suggested a causal role for Nrf2 activation in the upregulation of anti-oxidative enzymes by DJ-1. Therefore, the present study further investigated whether activation of Nrf2 is a prerequisite for DJ-1-mediated protection of $\mathrm{H} 9 \mathrm{c} 2$ cells against oxidative stress induced by H/R. To ascertain this question, H9c2 cells were transfected with pFlag-DJ-1 or empty vector pFlag for $24 \mathrm{~h}$ and 

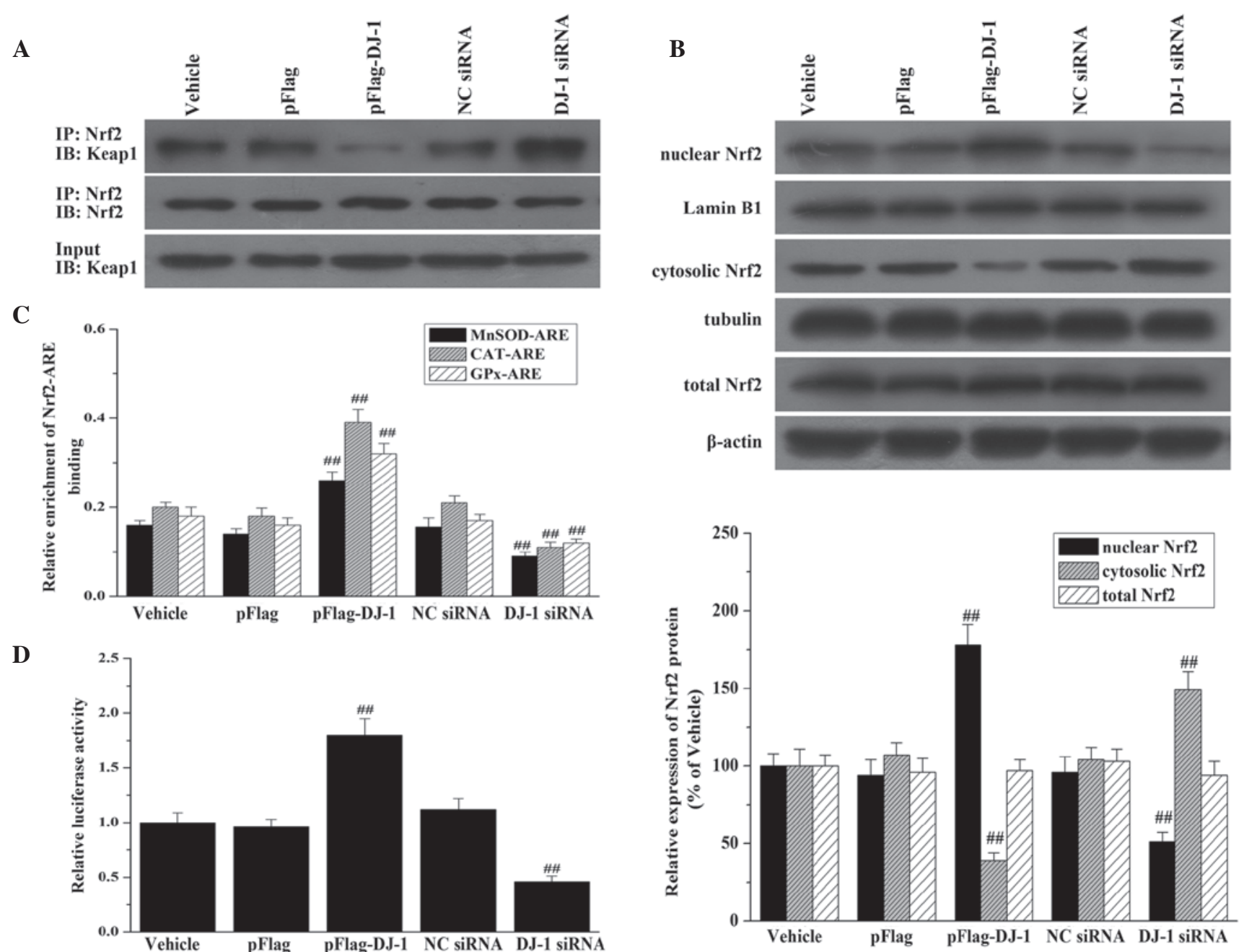

Figure 3. Role of DJ-1 in the regulation of the Keap1-Nrf2 interaction, Nrf2 nuclear translocation, ARE-binding and its transcriptional activity. H9c2 cells were transiently transfected with or without NC or Nrf2 siRNA, or pFlag-DJ-1 or its empty control vector pFlag for $24 \mathrm{~h}$. (A) Keap1-Nrf2 interaction was assessed by co-IP. Cell lysates were subjected to IP with anti-Nrf2 antibody and IB analysis was performed with anti-Keap1 or anti-Nrf2. Total protein extracts (prior to IP) were used as control (Input). Representative images of three separate experiments are shown. (B) Nrf2 nuclear translocation was assessed by western blot analysis. Whole-cell lysates, cytosolic and nuclear fractions were prepared and nuclear, cytosolic and total Nrf2 levels were examined. Lamin B1 was used as the nuclear marker and loading control, tubulin as the cytosolic marker and loading control, and $\beta$-actin as the whole-cell loading control. A representative blot of each experiment is shown with the densitometric analysis corresponding to the mean \pm standard error of the mean of four independent experiments. ${ }^{\# \#} \mathrm{P}<0.01$ vs. the corresponding vehicle group. (C) A chromatin IP assay was performed for the detection of ARE-binding activity of Nrf2. The relative binding of Nrf2 with ARE sequences in the promoter regions of MnSOD, CAT and GPx (MnSOD-ARE, CAT-ARE and GPx-ARE) were quantified from the band intensities of four independent experiments. Values are expressed as the mean \pm standard error of the mean. ${ }^{\# \#} \mathrm{P}<0.01$ vs. the corresponding vehicle group. (D) The transcriptional activity of Nrf2 was tested by luciferase reporter assay. H9c2 cells were co-transfected with the ARE reporter plasmid and the indicated siRNA or pFlag-DJ-1 for $24 \mathrm{~h}$ and were harvested for the luciferase assay. Relative luciferase activity was determined as firefly luciferase activity normalized to renilla luciferase activity. Values are expressed as the mean \pm standard error of the mean of triplicate experiments. ${ }^{\# \#} \mathrm{P}<0.01 \mathrm{vs}$. the corresponding vehicle group. siRNA, small interfering RNA; SOD, superoxide dismutase; GPx, glutathione peroxidase; CAT, catalase; NC, negative control; Nrf2, nuclear factor erythroid 2-like 2; Keap1, Kelch-like ECH-associated protein 1; ARE, anti-oxidant response element; IP, immunoprecipitation; IB, immunoblotting.

subsequently with NC or Nrf2 siRNA for $24 \mathrm{~h}$, followed by H/R. H/R-induced oxidative stress was analyzed by quantifying MDA and ROS content, and cellular damage was monitored by measuring cell viability and the release of LDH. As expected, DJ-1 overexpression protected H9c2 cells against H/R-mediated oxidative stress as shown by attenuation of H/R-induced viability loss (Fig. 5A), LDH release (Fig. 5B), as well as ROS (Fig. 5C) and MDA (Fig. 5D) content, whereas simultaneously knocking down Nrf2 significantly attenuated DJ-1-mediated cytoprotection. These results clearly indicated an essential role of Nrf2 activation for DJ-1-mediated cytoprotection against H/R-induced oxidative stress.

\section{Discussion}

The present study demonstrated that activation of the Nrf2 pathway is a critical mechanism by which DJ-1 upregulates anti-oxidative enzymes and attenuates H/R-induced oxidative stress in H9c2 cells. This was indicated by results showing that: 1) DJ-1 promoted Nrf2 and Keap1 dissociation, resulting in the enhanced levels of nuclear translocation of $\mathrm{Nrf} 2$ in $\mathrm{H} 9 \mathrm{c} 2$ cells; 2) DJ-1 increased the direct binding of Nrf2 to ARE on the promoters of MnSOD, GPx and CAT, and enhanced the transcriptional activity of Nrf2; 3) most importantly, knockdown of Nrf2 expression with siRNA not only prevented the induction of anti-oxidative enzymes (MnSOD, GPx and CAT) 

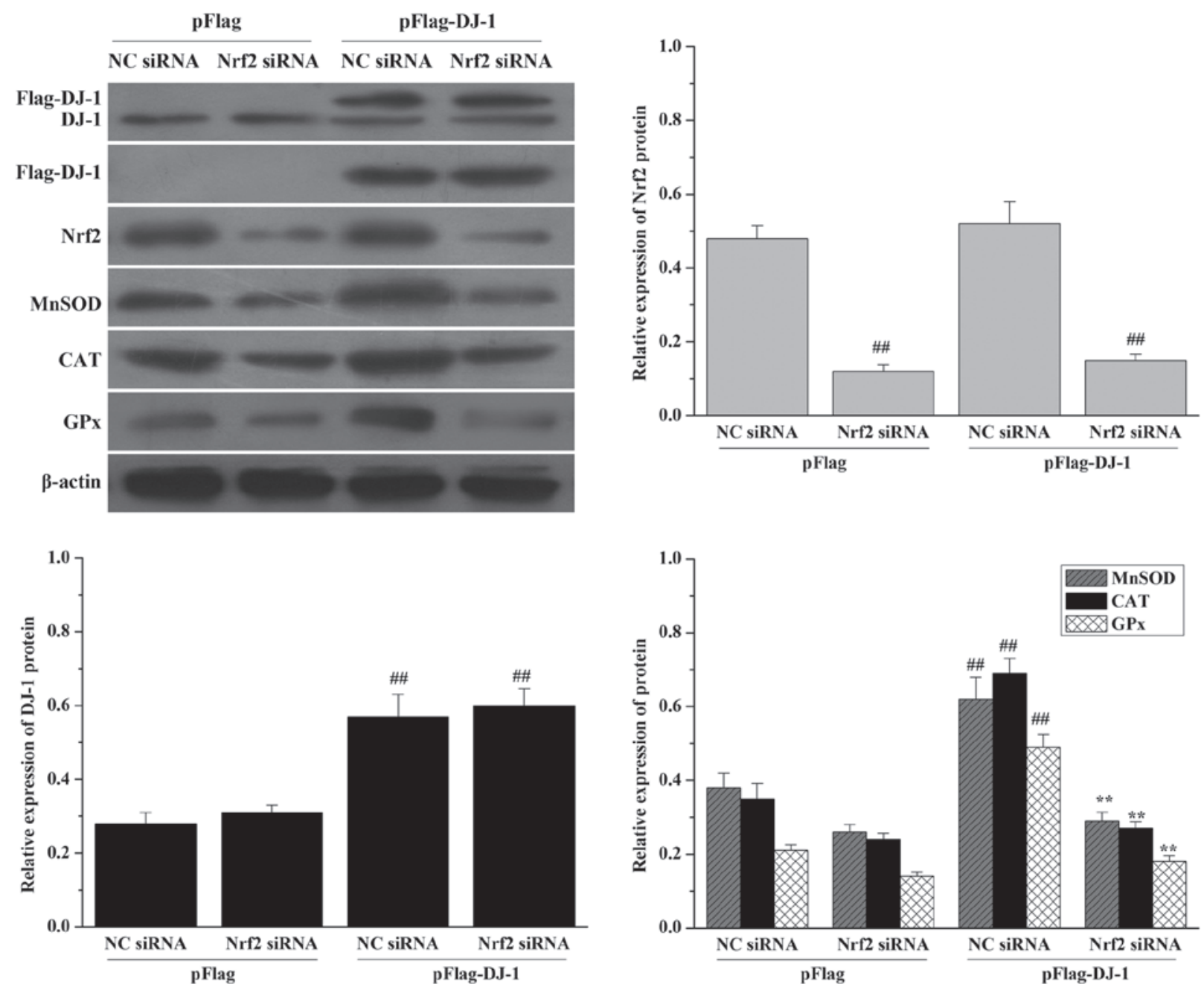

Figure 4. Effects of Nrf2 knockdown on the DJ-1-mediated induction of the anti-oxidant enzymes MnSOD, GPx and CAT in H9c2 cells. H9c2 cells were transfected with pFlag-DJ-1 or its empty vector pFlag for $24 \mathrm{~h}$ and subsequently with NC or Nrf2 siRNA for $24 \mathrm{~h}$. The overexpression of DJ-1 was detected by western blot analysis using DJ-1 and Flag antibodies, respectively. Levels of Nrf2 and the anti-oxidant enzymes MnSOD, GPx and CATs were examined with Nrf2, MnSOD, CAT and GPx antibodies. $\beta$-actin was used as an internal control. A representative blot of each experiment is shown with the densitometric analysis corresponding to the mean \pm standard error of the mean of four independent experiments. ${ }^{\# \prime} \mathrm{P}<0.01$ vs. the corresponding pFlag $+\mathrm{NC}$ siRNA group; ${ }^{* *} \mathrm{P}<0.01$ vs. the corresponding pFlag-DJ-1 + NC siRNA group. siRNA, small interfering RNA; SOD, superoxide dismutase; GPx, glutathione peroxidase; CAT, catalase; NC, negative control; Nrf2, nuclear factor erythroid 2-like 2.

by DJ-1 but also severely diminished the cardioprotective effects of DJ-1 against H/R-induced oxidative stress in H9c2 cells. In conclusion, the present study expanded the current understanding of the anti-oxidative stress function of DJ-1 by identifying a novel regulatory process induced by DJ-1, which stimulates the expression of anti-oxidative enzymes via activation of $\mathrm{Nrf} 2$ and, as a result, attenuates $\mathrm{H} / \mathrm{R}$-induced oxidative stress in $\mathrm{H} 9 \mathrm{c} 2$ cells.

Oxidative stress is directly associated with the pathogenesis of myocardial ischemia-reperfusion injury and is therefore an attractive target for biomarker development and potential therapies (20). DJ-1 is believed to have several possible functions, but a consistent finding is that it responds to oxidative stress. Several studies have demonstrated that DJ-1 is protective against Parkinson disease, ischemia-reperfusion and stroke-induced damage by attenuating oxidative stress (21-24). More recently, a study by our group investigated the potential role of DJ-1 in the response of H9c2 cells to oxidative stress induced by $\mathrm{H} / \mathrm{R}$ by using gene transfer strategies and identified that DJ-1-overexpressing H9c2 cells exhibited elevated levels of anti-oxidant enzymes, including MnSOD, CAT and GPx, and were more resistant to H/R-induced oxidative stress (11). Furthermore, the present study showed that DJ-1 knockdown suppressed the induction of these enzymes and further augmented the oxidative stress-induced injury, which was in agreement with the results for DJ-1 overexpressing cells. Again, these findings demonstrated that DJ-1 can induce anti-oxidant enzyme expression and attenuate H/R-induced oxidative stress in H9c2 cells. However, the mechanisms of the induction of these anti-oxidative enzymes by DJ-1 have remained elusive, and therefore, the present study focused on clarifying the underlying mechanisms.

Nrf2 is the primary transcription factor that mediates transcriptional regulation of numerous anti-oxidant genes (including MnSOD, CAT and GPx) by binding to the ARE element present in the promoters of these genes (16). Several studies have demonstrated that Nrf2 has a crucial role in the protection of cells against oxidative stress $(12,13)$. Of note, 
A

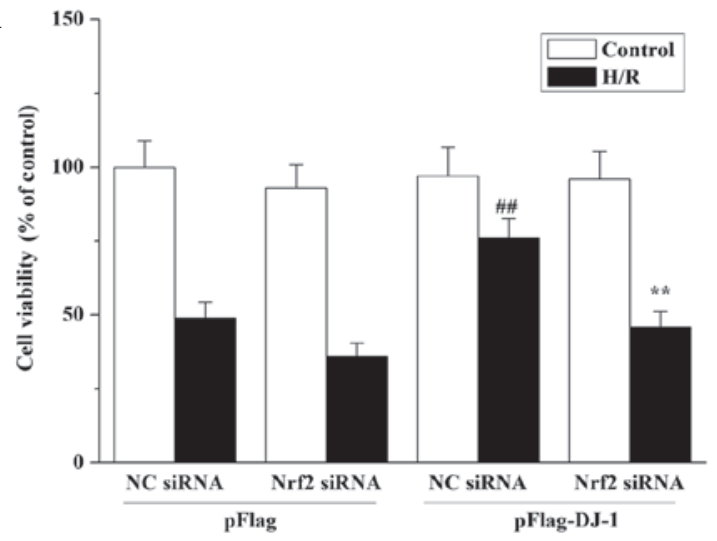

C

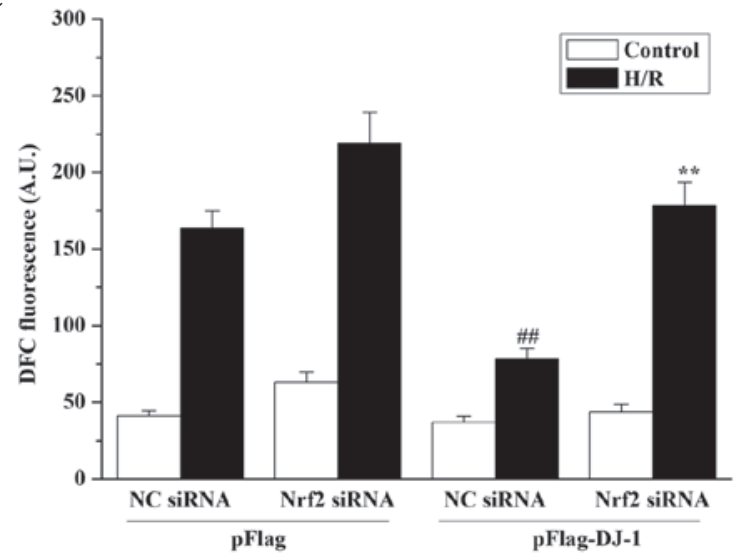

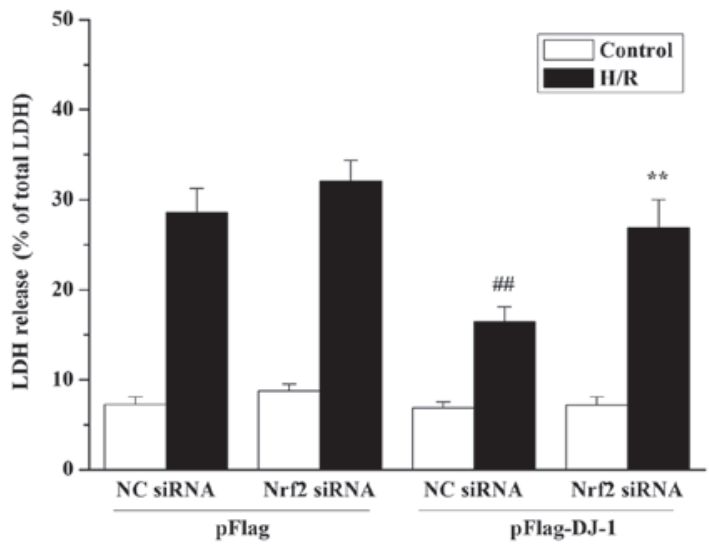

D

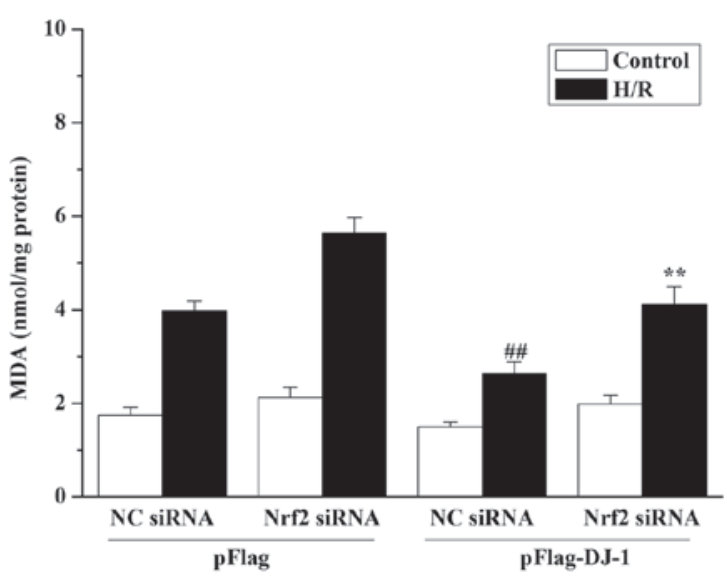

Figure 5. Effect of Nrf2 knockdown on DJ-1-mediated cytoprotection against H/R-induced oxidative stress in H9c2 cells. H9c2 cells were transfected with pFlag-DJ-1 or its empty vector pFlag for $24 \mathrm{~h}$ and subsequently with NC or Nrf2 siRNA for $24 \mathrm{~h}$ and subjected to H/R. Cellular damage was monitored by measuring (A) cell viability and (B) LDH release, and oxidative stress was analyzed by quantifying (C) ROS and (D) MDA content. Values are expressed as the mean \pm standard error of the mean of four independent experiments. ${ }^{\# \#} \mathrm{P}<0.01$ vs. the corresponding pFlag $+\mathrm{NC}$ siRNA $+\mathrm{H} / \mathrm{R}$ group; ${ }^{* *} \mathrm{P}<0.01 \mathrm{vs}$. the corresponding pFlag-DJ-1 + NC siRNA + H/R group. H/R, hypoxia/re-oxygenation; MDA, malondialdehyde; ROS, reactive oxygen species; LDH, lactate dehydrogenase; NC, negative control; siRNA, small interfering RNA; DFC, 2',7'-dichlorofluorescein.

DJ-1 has been reported to be a stabilizer and positive regulator of Nrf2, aiding Nrf2 in nuclear translocation and anti-oxidant gene activation $(18,25,26)$. On this basis, it was hypothesized that activation of the Nrf2 pathway may be responsible for the induction of anti-oxidative enzymes by DJ-1 and contribute to the protective functions of DJ-1 against H/R-induced oxidative stress in $\mathrm{H} 9 \mathrm{c} 2$ cells. To elucidate this question, the present study determined the effects of DJ-1 on the Keap1 - Nrf2 interaction and subsequent nuclear translocation, ARE-binding and transcriptional activity of $\mathrm{Nrf} 2$ in $\mathrm{H} 9 \mathrm{c} 2$ cells. The results showed that DJ-1 overexpression promoted Nrf2 - Keap1 dissociation and resulted in increased nuclear translocation, ARE-binding and transcriptional activity of Nrf2, whereas knockdown of DJ-1 exhibited adverse effects. These findings suggested that DJ-1 has a crucial role in the activation of the Nrf2 pathway in H9c2 cells. To further verify the contribution of the Nrf2 pathway in the DJ-1-mediated upregulation of anti-oxidative enzymes, the present study investigated the impact of Nrf2 knockdown on the induction of MnSOD, GPx and CAT by DJ-1. As expected, the results showed that DJ-1 overexpression led to a significant upregulation of MnSOD, GPx and CAT expression. These results are consistent with those of a previous study by our group (11). However, this effect was diminished in $\mathrm{H} 9 \mathrm{c} 2$ cells transfected with siRNA against Nrf2, suggesting that activation of the Nrf2 pathway is essential for the induction of anti-oxidative enzymes by DJ-1. In addition, considering the fact that the Nrf2 pathway has a critical role in cell survival under oxidative stress conditions, the present study further investigated whether activation of Nrf2 is a prerequisite for DJ-1-mediated protection of $\mathrm{H} 9 \mathrm{c} 2$ cells against oxidative stress induced by H/R. DJ-1 overexpression protected H9c2 cells against H/R-mediated oxidative stress as supported by the observed attenuation of H/R-induced loss in cell viability and LDH release, and elevation of ROS and MDA content. However, when Nrf2 expression was specifically knocked down using siRNA, the inhibitory effect of DJ-1 overexpression on H/R-mediated oxidative stress was reversed. These results clearly indicated an essential role of Nrf2 activation in DJ-1-mediated cytoprotection against $\mathrm{H} / \mathrm{R}$-induced oxidative stress.

However, of note, the question regarding the mechanism by which DJ-1 induces Nrf2 activation in H9c2 cells was not addressed by the present study and remains to be elucidated. Clements et al (18) reported that DJ-1 may activate the Nrf2 pathway by preventing the Keap1/Nrf2 association without binding to either component as well as by preventing the ubiquitination and proteasomal clearance of Nrf2. Similarly, the results of the present study confirmed that DJ-1 promotes 
only the Nrf2-Keap1 dissociation, as the co-IP assay did not indicate any binding between DJ-1 and either Nrf 2 or Keap1 in H9c2 cells (data no shown). However, to the best of our knowledge, no experimental evidence is available that DJ-1 prevents Nrf2 ubiquitination in this context. Therefore, it is concluded that DJ-1 is able to interact with other proteins to regulate Nrf2 stability and activation. In addition, previous studies reported that phosphorylation or acetylation of Nrf2 may regulate its intracellular localization and activity $(27,28)$. The possibility that DJ-1 may regulate the post-translational modification of Nrf2 cannot be excluded considering that DJ-1 interacts with transcriptional repressors, including protein inhibitor of activated signal transducer and activator of transcription, $2 \alpha$ and DJ-1-binding protein, and inhibits the recruitment of the histone deacetylase complex $(29,30)$.

In conclusion, the present study provided evidence that DJ-1 elicits significant Nrf2 activation and Nrf2-dependent induction of major cellular anti-oxidant enzymes and effectively attenuates $\mathrm{H} / \mathrm{R}$-induced oxidative stress in $\mathrm{H} 9 \mathrm{c} 2$ cells. These observations demonstrated the importance of the DJ-1/Nrf2 axis in the cellular response to oxidative stress-induced cell death and suggest that this axis may be a potential therapeutic target for cardiovascular disorders associated with oxidative stress such as myocardial ischemia-reperfusion injury.

\section{Acknowledgements}

The present study was supported by the Natural Scientific Foundation of China (grant nos. 81060022 and 81460060) and the Natural Scientific Foundation of Jiangxi Province (no. 2010GZY0220).

\section{References}

1. Dhalla NS, Temsah RM and Netticadan T: Role of oxidative stress in cardiovascular diseases. J Hypertens 18: 655-673, 2000.

2. Valko M,Leibfritz D, Moncol J, Cronin MT, Mazur M and Telser J: Free radicals and antioxidants in normal physiological functions and human disease. Int J Biochem Cell Biol 39: 44-84, 2007.

3. Marczin N, El-Habashi N, Hoare GS, Bundy RE and Yacoub M: Antioxidants in myocardial ischemia-reperfusion injury: Therapeutic potential and basic mechanisms. Arch Biochem Biophys 420: 222-236, 2003.

4. Venardos KM, Perkins A, Headrick J and Kaye DM: Myocardial ischemia-reperfusion injury, antioxidant enzyme systems and selenium: A review. Curr Med Chem 14: 1539-1549, 2007.

5. Nagakubo D, Taira T, Kitaura H, Ikeda M, Tamai K, Iguchi-Ariga SM and Ariga H: DJ-1, a novel oncogene which transforms mouse NIH3T3 cells in cooperation with ras. Biochem Biophys Res Commun 231: 509-513, 1997.

6. Ishikawa S, Taira T, Takahashi-Niki K, Niki T, Ariga H and Iguchi-Ariga SM: Human DJ-1-specific transcriptional activation of tyrosine hydroxylase gene. J Biol Chem 285: 39718-39731, 2010.

7. Shinbo Y, Taira T, Niki T, Iguchi-Ariga SM and Ariga H: DJ-1 restores p53 transcription activity inhibited by Topors/p53BP3. Int J Oncol 26: 641-648, 2005.

8. Taira T, Saito Y, Niki T, Iguchi-Ariga SM, Takahashi K and Ariga H: DJ-1 has a role in antioxidative stress to prevent cell death. EMBO Rep 5: 213-218, 2004.

9. Shendelman S, Jonason A, Martinat C, Leete T and Abeliovich A: DJ-1 is a redox-dependent molecular chaperone that inhibits alpha-synuclein aggregate formation. PLoS Biol 2: e362, 2004.

10. Honbou K, Suzuki NN, Horiuchi M, Niki T, Taira T, Ariga H and Inagaki F: The crystal structure of DJ-1, a protein related to male fertility and Parkinson's disease. J Biol Chem 278: 31380-31384, 2003.
11. Yu HH, Xu Q, Chen HP, Wang S, Huang XS, Huang QR and He M: Stable overexpression of DJ-1 protects H9c2 cells against oxidative stress under a hypoxia condition. Cell Biochem Funct 31: 643-651, 2013.

12. Kaspar JW, Niture SK and Jaiswal AK: Nrf2: INrf2 (Keap1) signaling in oxidative stress. Free Radic Biol Med 47: 1304-1309, 2009.

13. Nguyen T, Nioi P and Pickett CB: The Nrf2-antioxidant response element signaling pathway and its activation by oxidative stress. J Biol Chem 284: 13291-13295, 2009.

14. Keum YS and Choi BY: Molecular and chemical regulation of the Keap1-Nrf2 signaling pathway. Molecules 19: 10074-10089, 2014.

15. Sun Z, Zhang S, Chan JY and Zhang DD: Keap1 controls postinduction repression of the Nrf2-mediated antioxidant response by escorting nuclear export of Nrf2. Mol Cell Biol 27: 6334-6349, 2007.

16. Howden R: Nrf2 and cardiovascular defense. Oxid Med Cell Longev 2013: 104308, 2013.

17. Lee JM and Johnson JA: An important role of Nrf2-ARE pathway in the cellular defense mechanism. J Biochem Mol Biol 37: 139-143, 2004.

18. Clements CM, McNally RS, Conti BJ, Mak TW and Ting JP: DJ-1, a cancer- and Parkinson's disease-associated protein, stabilizes the antioxidant transcriptional master regulator Nrf2. Proc Natl Acad Sci USA 103: 15091-15096, 2006.

19. Huang XS, Chen HP, Yu HH, Yan YF, Liao ZP and Huang QR: Nrf2-dependent upregulation of antioxidative enzymes: A novel pathway for hypoxic preconditioning-mediated delayed cardioprotection. Mol Cell Biochem 385: 33-41, 2014.

20. Perrelli MG, Pagliaro P and Penna C: Ischemia/reperfusion injury and cardioprotective mechanisms: Role of mitochondria and reactive oxygen species. World J Cardiol 3: 186-200, 2011.

21. Dongworth RK, Mukherjee UA, Hall AR, Astin R, Ong SB, Yao Z, Dyson A, Szabadkai G, Davidson SM, Yellon DM, et al: DJ-1 protects against cell death following acute cardiac ischemia-reperfusion injury. Cell Death Dis 5: e1082, 2014.

22. Yanagisawa D, Kitamura Y, Inden M, Takata K, Taniguchi T, Morikawa S, Morita M, Inubushi T, Tooyama I, Taira T, et al: DJ-1 protects against neurodegeneration caused by focal cerebral ischemia and reperfusion in rats. J Cereb Blood Flow Metab 28: 563-578, 2008.

23. Inden M, Taira T, Kitamura Y, Yanagida T, Tsuchiya D, Takata K, Yanagisawa D, Nishimura K, Taniguchi T, Kiso Y, et al: PARK7 DJ-1 protects against degeneration of nigral dopaminergic neurons in Parkinson's disease rat model. Neurobiol Dis 24: 144-158, 2006.

24. Aleyasin H, Rousseaux MW, Phillips M, Kim RH, Bland RJ, Callaghan S, Slack RS, During MJ, Mak TW and Park DS: The Parkinson's disease gene DJ-1 is also a key regulator of stroke-induced damage. Proc Natl Acad Sci USA 104: 18748-18753, 2007.

25. Malhotra D, Thimmulappa R, Navas-Acien A, Sandford A, Elliott M, Singh A, Chen L, Zhuang X, Hogg J, Pare P, et al: Expression of concern: Decline in NRF2-regulated antioxidants in chronic obstructive pulmonary disease lungs due to loss of its positive regulator, DJ-1. Am J Respir Crit Care Med 178: 592-604, 2008

26. Ustariz CR, Arce S, Hernández P, Almaguer M, Pérez S and Ballester JM: HLA antigens in paroxysmal nocturnal hemoglobinuria. Acta Haematol 63: 52-54, 1980.

27. Sun Z, Chin YE and Zhang DD: Acetylation of Nrf2 by p300/CBP augments promoter-specific DNA binding of Nrf2 during the antioxidant response. Mol Cell Biol 29: 2658-2672, 2009.

28. Sun Z, Huang Z and Zhang DD: Phosphorylation of Nrf2 at multiple sites by MAP kinases has a limited contribution in modulating the Nrf2-dependent antioxidant response. PLoS One 4: e6588, 2009.

29. Niki T, Takahashi-Niki K, Taira T, Iguchi-Ariga SM and Ariga H: DJBP: A novel DJ-1-binding protein, negatively regulates the androgen receptor by recruiting histone deacetylase complex and DJ-1 antagonizes this inhibition by abrogation of this complex. Mol Cancer Res 1: 247-261, 2003.

30. Takahashi K, Taira T, Niki T, Seino C, Iguchi-Ariga SM and Ariga H: DJ-1 positively regulates the androgen receptor by impairing the binding of PIASx alpha to the receptor. J Biol Chem 276: 37556-37563, 2001. 